\title{
AN EXTENDED LATIN HYPERCUBE SAMPLING APPROACH FOR CAD MODEL GENERATION
}

\author{
Shahroz KHAN ${ }^{1,}$, Erkan GÜNPINAR ${ }^{1}$ \\ ${ }^{1}$ Smart CAD Laboratory, School of Mechanical Engineering, İstanbul Technical University, İstanbul, Turkey
}

\begin{abstract}
In this paper, extended version of Latin hypercube sampling (ELHS) is proposed to obtain different design variations of a CAD model. The model is first represented by design parameters. Design constraints that are relationships between the parameters are then determined. After assigning value ranges for the design parameters, design space is formed. Each design parameter represents a dimension of this design space. Design is a point in the design space and is feasible if it satisfies the predefined design constraints. Otherwise, it is infeasible. ELHS utilizes an input design in order to obtain feasible designs.

All dimensions of the design space are divided into equal number of intervals. ELHS perform trials in design space to find feasible designs. In each trial, all the candidate designs are enumerated and one of them is selected based on a cost function. Value of the cost function is zero if all design constraints of the design are satisfied. A similarity constraint is introduced in order to eliminate designs with similar geometries. Three different CAD models are utilized for this study's experiments in order to show the results of the ELHS algorithm.
\end{abstract}

Keywords: Latin hypercube sampling, Computer aided design, Geometrical designs, Constrained design space

\section{INTRODUCTION}

Engineering and industrial product design is a goal oriented, constrained based and decision making process. The product obtained after this process should satisfy consumer's needs not just by functional performance also by external appearance. Design process can be more complex and time consuming if the appearance of the product is valuable to its consumers. Therefore, a tool which can automatically generates variety of design options for a product within product's design space can be beneficial. This can provide designers different options for the selection of the most appealing design. The objective of this research is to develop a technique that can produce variety of design options for a product within the designer's design requirements. For this, a sampling technique called Latin Hypercube sampling (LHS) is selected and modified according to the objectives of the current research.

LHS is a popular stratified sampling technique which was first proposed by MacKay et. al. [1] and was further improved by Iman and Conover [2]. It is a method of sampling random designs that attempts to distribute evenly in the design space. In this research, traditional LHS technique is extended to perform sampling in the constrained and high dimensional design spaces, which is named as Extended Latin Hypercube sampling (ELHS). The CAD model is defined by design parameters. Relationships between these parameters are also determined and are called design constraints. Range of each design parameter is specified by defining its lower and upper bounds. These design parameters and their bounds form the design space in which sampling is performed. Each design parameter represents a dimension in the design space and sampling is performed by dividing the all dimensions into equally spaced number of intervals (levels). This division partitioned the design space into sub-spaces (stratums).

During the search process in the design space, ELHS performs equal number of trials as the number of intervals of the design space. Only one design is selected from each interval. There can be many feasible

*Corresponding Author: shahrozkhan2020@gmail.com 
and infeasible designs in the dimension interval. In order to select a feasible one, enumeration is performed during the trails. All the candidate designs are enumerated in each trial and the one which minimizes the cost is selected. The cost function is defined based on the equality and inequality design constraints. A similarity constraint is applied based on the Euclidian distance formulation which ensures the generation of designs with variant geometries. Figure 1 shows designs generated using proposed methodology. A vase model is given as input and different designs are obtained. We believe that such automatic generation of CAD models can be very useful for design engineers and inspire them in the design process.
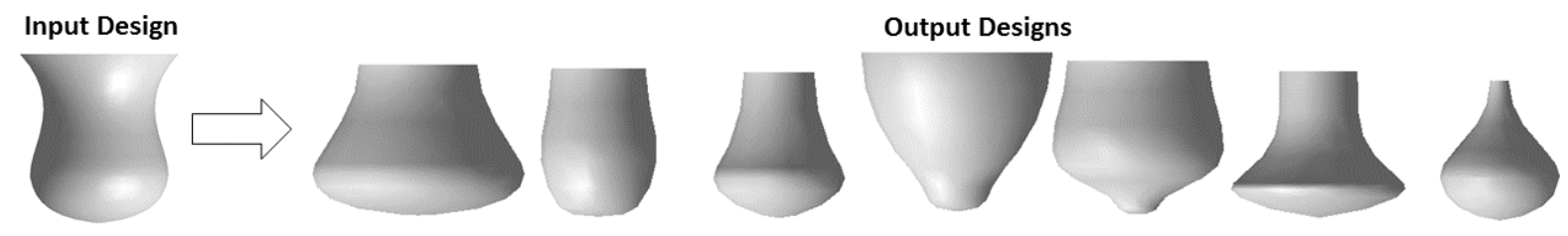

Figure 1. Variant shapes of vase model obtained by the proposed methodology

\section{RELATED WORKS}

Several improvements have been done by different researcher in order to improve LHS sampling. The method to perform uniform sampling in multidimensional space for LHS was introduced by Johnson et al. [3]. In this method, designs to be sampled using LHS are obtained while maximizing the minimum distance between designs (maximin criterion). The algorithm starts with a random design in the design space and searches for the next design with maximum of the minimum (maximin) inter-design distance. The maximin criterion was also used by Morris and Mitchell [4]. They used a simulated annealing search algorithm to search design space for designs which offer a compromise between the entropy and maximin criterion. Deutsch et al. [5] improved the method of maximin criterion by using Cholesky decomposition of correlation matrix and compared the proposed algorithm with simple LHS and Monte Carlo simulation. The obtained results showed that samples produced by their algorithm have better uniformity in the design space.

An algorithm for the best space filling designs was proposed by the Cioppa and Lucas [6]. However, their algorithm is computationally expensive because it requires the long run times. A Sliced Latin Hypercube (SLH) technique was introduced by Qian [7]. Design space is further sub-divided small design spaces and then sampling is performed in the sub-divided design spaces. This sub-division improves the space filling property of original LHS. SLH was further improved by Cao and liu [8]. They proposed a method which optimizes the sub-divided design space in order to maintain uniformity. Prescott [9] performed complete or partial enumeration searches to investigate the space-filling properties of orthogonal-column Latin Hypercube designs with multiple number of runs. He used the maximin criterion in cases where there are several designs with similar properties.

There are several other approaches proposed for the optimal distribution of designs in design space. Sacks et. al. [10] introduced a method of minimization of the integrated mean square error (IMSE). Shewry and Wynn performed selection of designs based on the maximization of entropy. Bates et. al. $[11,12]$ used approach of minimization of potential energy of designs according to Audze and Eglais [13]. Rajabi et. al. [14] studied the impact of initial design feed to LHS. They compared the effect of design selections randomly from the stratums and the selection of designs from the mid-point of stratums. The results revealed that design selection based on mid-point is significantly better than those based on random selection.

Several other variations of LHS based on orthogonality of design space are also proposed by different researchers. Leary et. al. [15] introduced orthogonal-array-based LHS designs, Joseph and Hung [16] proposed orthogonal-maximum LHS designs and orthogonal and nearly orthogonal designs was 
demonstrated by Bingham et. al. [17]. There is considerable amount of research has been done on optimal selection of designs in the design space in order to improve the space-filling property of LHS. However, most works done by researchers are proposed for the unconstrained design spaces. The research problem becomes very complicated when selection of designs has to be performed in a constrained and high dimensional design space, as that of the current research. Mysakova et al. [18] and Mysakova and Leps [19] proposed a technique to perform sampling for constrained spaces. This technique is based on the triangulation of admissible space by Delaunay Triangulation method. To produce uniform sampling in the design space, a heuristic method based on the uniform finite element meshes was proposed. Although this technique has good space filling property but it is just applicable to only two dimensional constraint problems. Fuerle and Sienz [20] proposed a method based on LHS for design selections for constrained spaces. Desired number of designs to be sampled are defined first and these points are then randomly sampled using LHS in two dimensional space. The coordinates of samples that are in infeasible region are modified using the mutation operator that is used for the genetic algorithms. Fuerle and Sienz method has some draw backs such that it cannot be implemented for the high dimensional sampling problems more than 3D. Furthermore, it does not produce good results for the designs space where infeasible designs are spread irregularly.

However, ELHS has the ability to perform sampling in more than three-dimension design spaces. ELHS also ensures the selection of variant feasible designs from the design spaces in which the distribution of the infeasible designs is highly irregular.

\section{RESEARCH PROBLEM}

LHS is a stratified sampling technique in which random samples of design can be generated from a multidimensional design space. Suppose an $j$-dimensional design space and we want to sample $j$ number of designs evenly throughout the design space. The design parameters forms a specific geometrical design (or design) and are denoted by $x_{1}, x_{2}, x_{3}, \ldots, x_{j}$. Each design parameter defines a dimension in the design space. The value of each design parameter ranges between its lower and upper bound $\left[x_{n}^{l}, x_{n}^{u}\right]$ where $n=1,2,3, \ldots, j, x_{n}^{l}$ and $x_{n}^{u}$ are the lower and upper bounds of the $n^{\text {th }}$ design parameter, respectively. The range of each design parameter is partitioned into $N+1$ equally spaced number of intervals (levels) such that $\left[x_{n}^{l}=x_{n}^{1}, x_{n}^{2}, x_{n}^{3}, \ldots \ldots, x_{n}^{N}, x_{n}^{N+1}=x_{n}^{u}\right]$, where $N$ is the sampling scale. The $N+1$ number of partitions of each dimension divides the design space into $N^{j}$ number of sub-spaces (stratums). LHS samples the designs from the stratums of design space and creates a design array $(T)$ which contain the sampled designs (see Figure 2 (c)).

During the search process of LHS to generate design array $T, t$ is the number of trials that are performed on the basis of $L H$-rule and $t=N$. The LH-rule states that in any trial one and only one design can be selected from each interval of the design space. In the other trials, designs cannot be selected from a stratum of interval which is contiguous to the previous selected stratum (as case in Figure 2 (a)). For example, there are two designs (mark in black) are selected in the first trial as seen in the upper image of Figure 2 (a) which violated the LH-rule. During the second trial, a design is selected from the stratum that is contiguous to the previously selected stratum during trial-1 (see the lower image of Figure 2 (a)). This also violated the LH-rule. The Figure 2 (b) shows the sampling in the two dimensional design space satisfying the LH-rule.

There are feasible and infeasible designs in the constrained design space. The feasible design is one which satisfy the all geometrical constraints $\varphi_{1}, \varphi_{2}, \varphi_{3}, \ldots, \varphi_{m}$ which define relationships between the design parameters and $m$ is integer. Infeasible design is one which does not satisfy at least one of the geometrical constraints. The design constraints in this work can be equality or inequality constraints. As LHS selects the designs randomly in the design space, selected designs can be infeasible. Therefore, random selection nature of LHS is modified in the current research. A systematic method is proposed 
for the selection of feasible designs based on LHS and for higher dimensional design space with equality and inequality constraints.

(a)
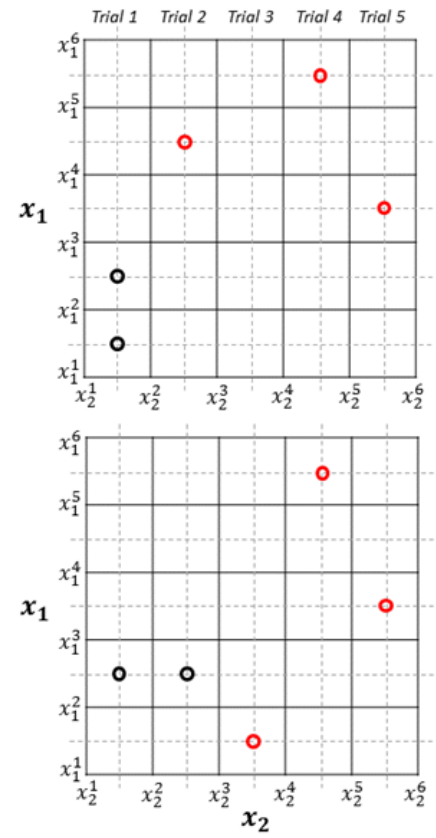

(b)

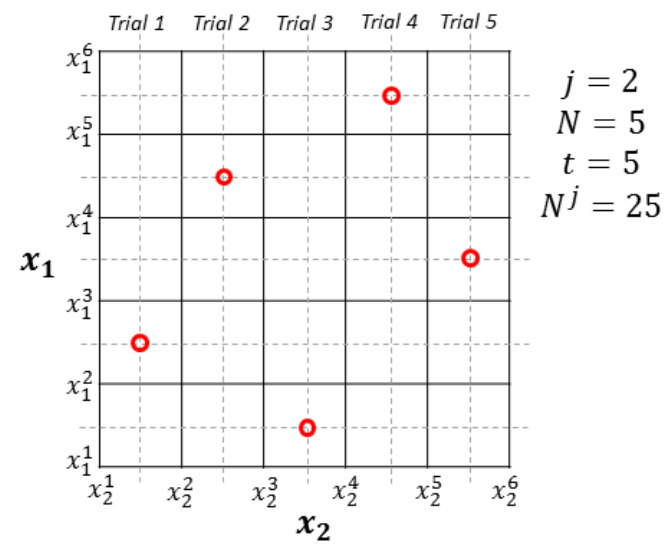

(c)

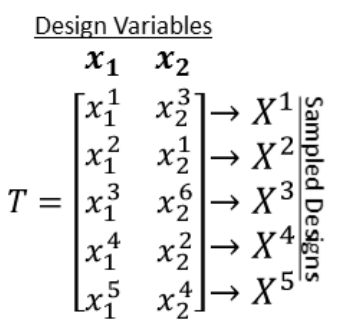

Figure 2. Implementation of LHS in the two dimensional design space with the sampling scale $N=5$. The red circle in the design space represents the sampled design. (a) Sampling is done while violating the LH-rule. (b) Sampling is done while satisfying the LH-rule. (c) Design array generated from the LHS implemented in image (b).

\section{METHODOLOGY}

The developed method gets a feasible design and its design space as input. Recall that a design space is formed using design parameters, design constraints and lower/upper bounds of each design parameter. Depending on the number of design to be sampled, $N$ (sampling scale) is also given. ELHS starts sampling the designs by performing trials in the design space. During a trial, candidate positions for each design parameter are enumerated and the candidate position having minimum cost value and satisfying the LH-rule is selected.

To obtain designs with distinct geometries, a similarity threshold $\mu$ is utilized. During each trial, at most a feasible design is obtained and its distance to other previously selected designs should be greater than $\mu$. By doing this, we can store designs distinct from the previously obtained ones. Euclidean distance is utilized to compute distance between two designs $p$ and $q$ and calculated as follows: $D_{p q}=$ $\sqrt{\sum_{n=1}^{j}|| x_{n}^{p}-\left.x_{n}^{q}\right|^{2}} \cdot x_{n}^{p}$ and $x_{n}^{q}$ represents scaled parameter values for the designs $p$ and $q$ in the $j$-th dimension of the design space. The scaled parameter values are obtained after normalizing the parameter values between 0 and 1 . These scaled parameter values forms the scaled design space. If all distances between the newly selected design and other previously obtained designs are greater than $\mu$, then newly selected design is stored. Otherwise it is rejected.

Below pseudo-code summarizes the ELHS algorithm. 
ELHS Algorithm:

1: //Input: initial feasible design $X^{\text {initial }}:\left[\begin{array}{lll}x_{1} & \ldots & x_{j}\end{array}\right]$, lower and upper bounds $\left[x_{n}^{l}, x_{n}^{u}\right]$ of each design parameter

2: Set the first design as $X^{1}=X^{\text {initial }}$

3: for $t=1$ to $N$ do

4: $\quad$ Set $x_{1}$ equal to $t$

5: $\quad$ for $n=2$ to $j$ do

6: $\quad$ for $p=1$ to $N$ do

7: $\quad$ Set $x_{n}=p$

8: $\quad$ Calculate cost value for design $X^{t}$

9: $\quad$ if cost value $=0$ and LH-rule satisfied then

10: $\quad$ Preserve $p$ for $x_{n}$

11: $\quad$ else

12: $\quad$ Reject $p$

13: $\quad$ end else

14: $\quad$ end if

15: end for

16: end for

17: $\quad / / h$ is total number of stored designs

18: $\quad$ for $k=1$ to $h$

19: $\quad$ Calculate distance $D_{t q}$ between $X^{t}$ and the $k^{t h}$ stored design $X^{q}$

20: $\quad$ if $D_{t q}>\mu$ then

21: $\quad$ Insert $X^{t}$ into the design array $T$

22: $\quad$ else

23: $\quad$ Reject $X^{t}$

24: $\quad$ end else

25: $\quad$ end if

26: end for

27: end for

Here we illustrate the ELHS algorithm for a 3-dimensional design space. Therefore, design variables are denoted by $x_{1}, x_{2}$ and $x_{3}$ representing each dimension of the design space (see Figure 3 ). Each dimension is divided into $N+1$ divisions, where $N=5$. There are 125 stratums $\left(N^{j}=125\right)$ and five trials will be performed $(t=5)$. Suppose the parameter set of initial input feasible design is

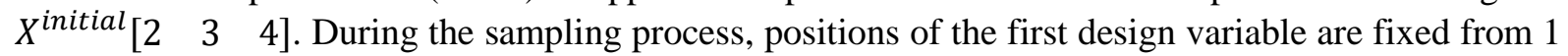
to 5 in order to decrease the total number of enumerations (see Figure 3 (a)). Using the input parameter set in the first trial $x_{2}$ is enumerated for its available candidate positions $1,2, \ldots, N$. During this enumeration, position of $x_{1}$ is set equal to 1 and position of $x_{3}$ to 4 which is selected from input parameter set. On the basis of cost function, $x_{2}=2$ is selected (see Figure 3 (a)). Proceeding to $x_{3}$, number of available candidate positions for the design parameter $x_{3}$ are $1,2, \ldots, N$. Enumeration is performed for $x_{3}$ between its candidate positions while taking $x_{1}=1$ and $x_{2}=2$. During this enumeration $x_{3}=3$ is selected on this basis of the cost function. 
(a)

\begin{tabular}{|c|c|c|c|c|c|}
\hline \multirow{12}{*}{$\begin{array}{c}j=3 \\
N=5 \\
t=5 \\
N^{j}=125\end{array}$} & $\begin{array}{c}\text { Design } \\
\text { Variables }\end{array}$ & $x_{1}$ & $x_{2}$ & $x_{3}$ & \multirow{2}{*}{$\begin{array}{l}\text { Candidate solution } \\
\text { variables during } \\
\longrightarrow 1,2,3, \ldots, N\end{array}$} \\
\hline & $\begin{array}{l}\text { Initial } \\
\text { Design }\end{array}$ & 2 & 3 & 4 & \\
\hline & \multirow{2}{*}{ Trial 1} & 1 & 2 & $\rightarrow \underline{4}$ & \multirow{3}{*}{$\begin{array}{l}\rightarrow 1,2,3, \ldots, N \\
\rightarrow 1,3,4,5\end{array}$} \\
\hline & & 1 & $\underline{2}$ & 3 & \\
\hline & \multirow{2}{*}{ Trial 2} & 2 & 4 & $\underline{3}$ & \\
\hline & & 2 & $\rightarrow \underline{4}$ & 2 & \multirow{2}{*}{$\begin{array}{l}\longrightarrow \\
\longrightarrow \\
\longrightarrow\end{array}$} \\
\hline & \multirow{2}{*}{ Trial 3} & 3 & 1 & $\underline{2}$ & \\
\hline & & 3 & $\rightarrow 1$ & 5 & \multirow{3}{*}{$\begin{array}{l}\rightarrow \\
\longrightarrow\end{array}$} \\
\hline & \multirow{2}{*}{ Trial 4} & 4 & 5 & $\underline{5}$ & \\
\hline & & 4 & $\Rightarrow 5$ & 1 & \\
\hline & \multirow{2}{*}{ Trial 5} & 5 & 3 & $\underline{1}$ & \multirow{2}{*}{$\begin{array}{l}\longrightarrow \\
\longrightarrow\end{array}$} \\
\hline & & 5 & -3 & 4 & \\
\hline
\end{tabular}

(b)

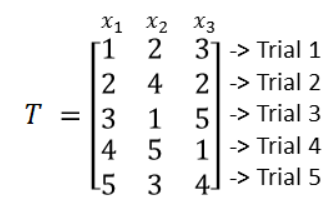

(c) Sampled Designs in 3D Design Space

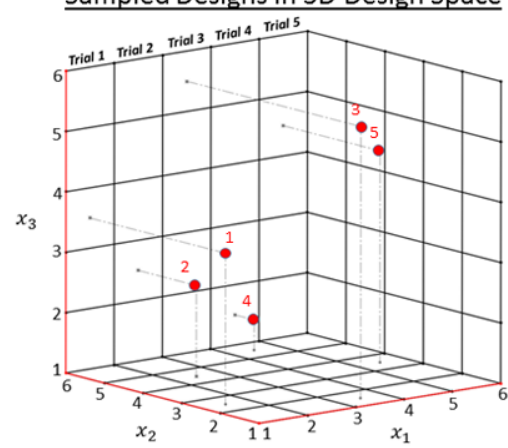

Figure 3. (a) Selection of candidate position during the trials. In which candidate position which minimizes the cost function is selected. (b) Design array containing the design. (c) 3-D plot of sampled designs.

In the second trial, $x_{1}$ is set to 2 and enumeration is performed for $x_{2}$ between its candidate positions $1,3,4,5$. According to LH-rule, as 2 is already selected for $x_{2}$ in the first trial, it cannot be selected in the current trial. Therefore, 2 is eliminated from the candidate positions of $x_{2}$ in second trial to validate the LH-rule. In this trial, the candidate positions for $x_{3}$ are $1,2,4,5$. The position 3 is eliminated to validate the LH-rule, as it is selected in the first trial. Accordingly, the selection is done for the next three trials. Notice that in Figure 3 (a), number of candidate positions for design variables decreases as the number of trial increases because of the LH-rule. In the proposed technique, sampling process starts

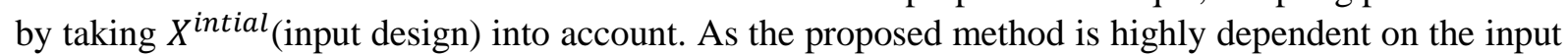
design, different inputs will apparently produce different designs.

\subsection{Cost Function}

Selection of designs during enumeration is done based on the cost function $C_{f}$ consisting of both equality and inequality design constraints. During each trial, cost value is computed for all the candidate positions of a design parameter. The one which minimizes the cost function is selected. The cost function is formulated as follows:

$$
C_{f}=\sum_{m=1}^{l} G\left(\varphi_{m}\right)
$$

$\varphi_{m}$ represents a design constraint and $G\left(\varphi_{m}\right)$ is the penalty function to penalize the design parameter value that does not satisfy the constraint. Let $F\left(\varphi_{m}\right)$ represents the equation of the design constraint $\varphi_{m}$ which can be either 0 or greater/smaller then 0 for equality or inequality constraints, respectively. $G\left(\varphi_{m}\right)$ gets 0 if the equality or inequality constraint is satisfied. Otherwise, it is absolute value of $F\left(\varphi_{m}\right)$ (see Equation 2). With such formulation, $C_{f}$ is 0 for feasible designs and positive for infeasible ones.

$$
\begin{aligned}
& G\left(\varphi_{m}\right)=0 \quad \text { if } \quad \varphi_{m} \text { satisfied } \\
& G\left(\varphi_{m}\right)=\left|F\left(\varphi_{m}\right)\right| \text { if } \varphi_{m} \text { not satisfied }
\end{aligned}
$$




\subsection{Multiple Run of the ELHS Algorithm}

The ELHS algorithm is executed multiple times to increase the number of designs obtained. Number of designs obtained may not be equal to number of divisions $N$ after applying the ELHS algorithm. The algorithm runs multiple times until no design with distinct geometries are produced. The proposed method is dependent on the input design as previously stated. Different designs can be obtained by feeding different input designs to the ELHS algorithm.

Figure 4 illustrates the usage scenario of the ELHS algorithm. In the first run, the input design is the initial feasible design $\left(X^{\text {intial }}\right)$ provided by the designer. The output of the first run is two variant feasible designs. In the second run, input to the ELHS algorithm-1 is the first design obtained from the first run. In third run, input is the second design obtained during the first run. Accordingly, multiple runs are performed until no distinct input design is obtained. Recall that distinctness is computed based on the similarity threshold $\mu$ in Section 4 .

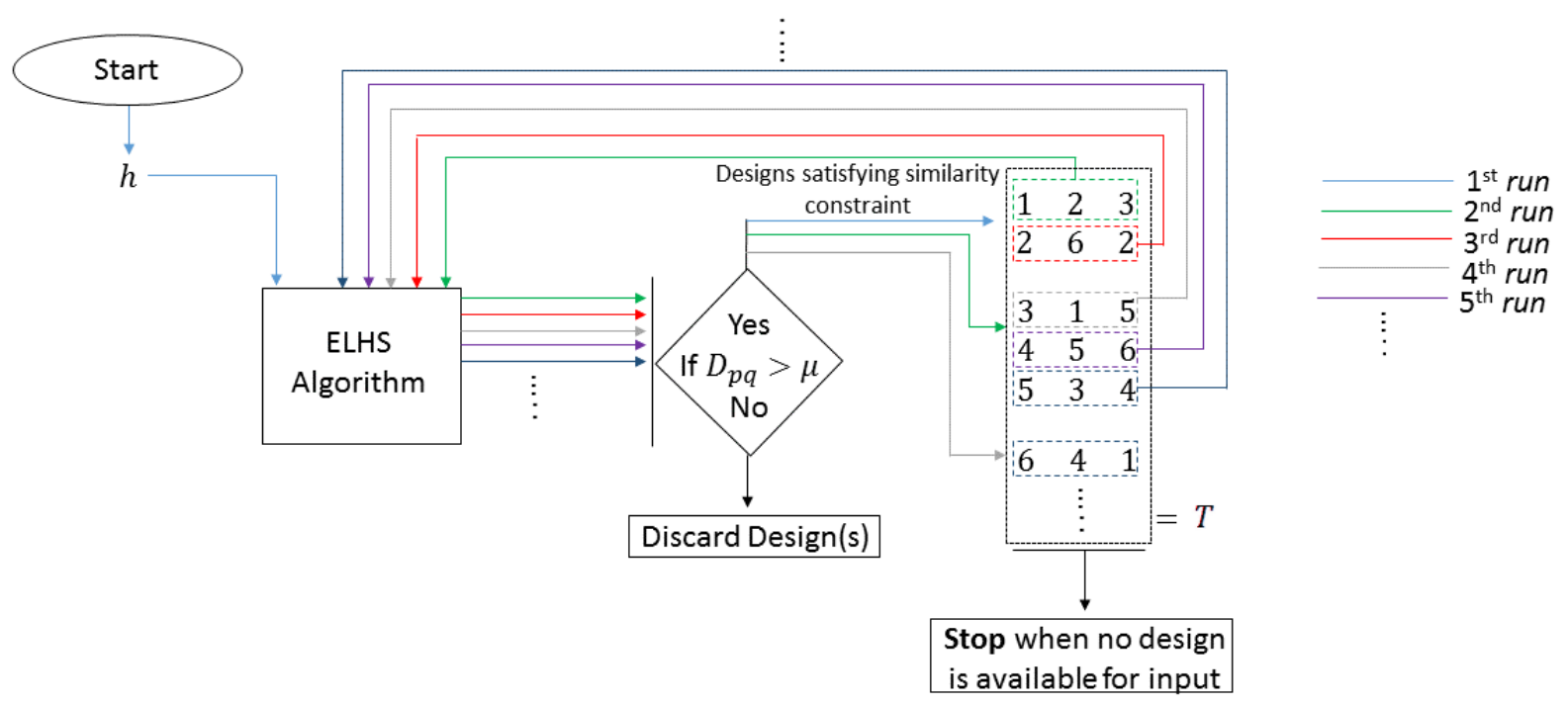

Figure 4. Usage scenario of the ELHS algorithm 


\section{EXPERIMENTAL STUDIES}

Several experiments have been conducted to check the efficiency of the proposed technique. Three different CAD models are selected as test models as seen Figure 5: Vase, glass and bowl.
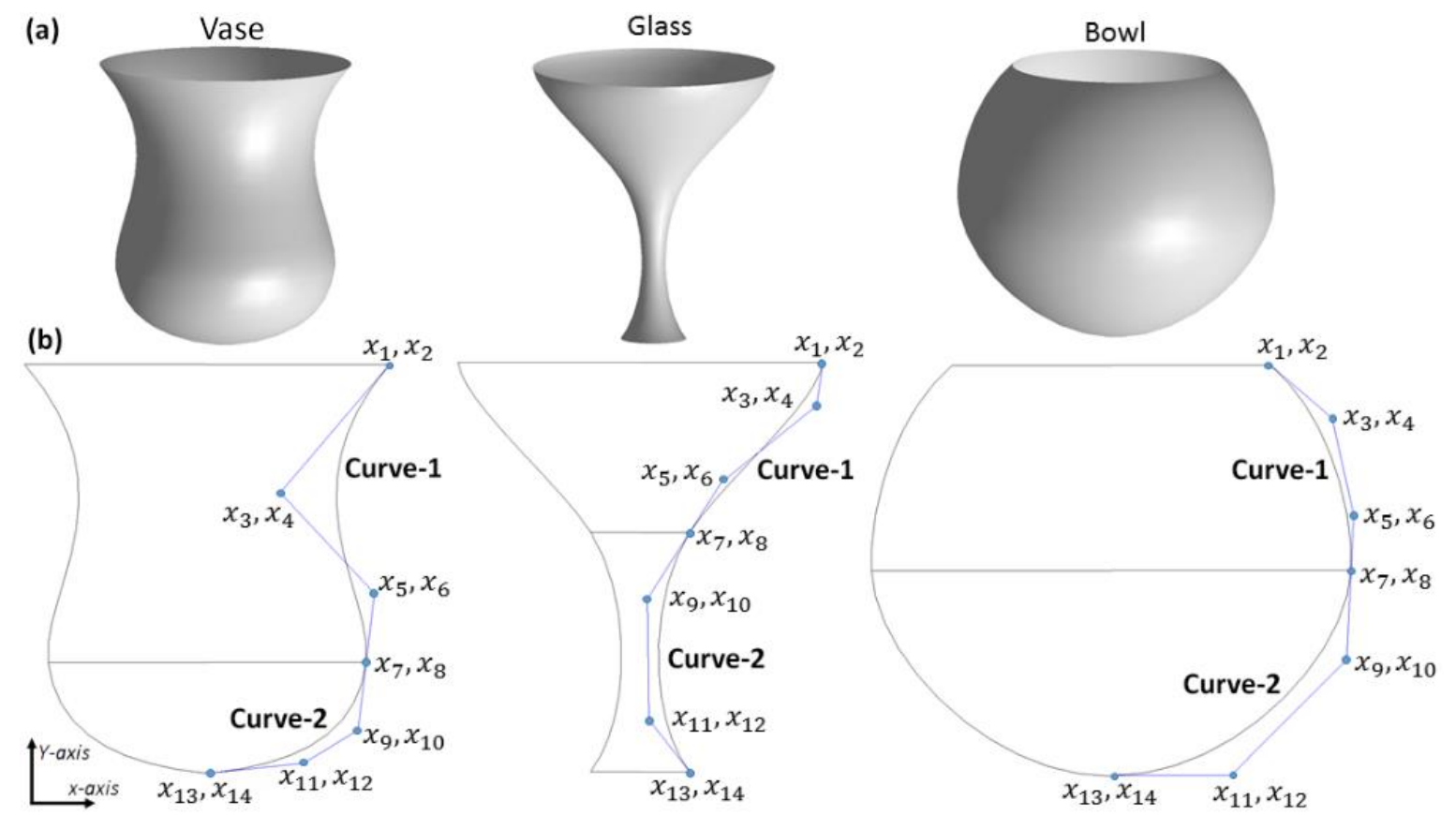

Figure 5. Selected input CAD models to the proposed technique. (a) Surface models. (b) 2D wireframe models

\subsection{Design Parameters}

Number of design parameters and design constraints are kept same for the all three designs in order to verify and to gain better perspective of the behavior of the proposed method under the same design space. Each input design is composed of two cubic Bezier curves in X-Y plane and consists of fourteen design parameters (see Figure 5(b)). 3-D surfaces for these models are created by performing the revolve operation on the cubic Bezier curves around $Y$-axis. For better visual appearance, $G^{0}$ and $G^{1}$ continuity is maintained at the connection of curve- 1 and curve- 2 of all three input designs. Design parameters $\left(x_{1}, x_{2}, x_{3}, \ldots, x_{14}\right)$ are defined on the control points of curves as shown in Figure 5 (b). The design parameters with odd integer numbered subscript represents the $X$-coordinate and design parameters with even integer numbered subscript represents the $Y$-coordinate of control points. For example, $x_{1}$ and $x_{2}$ are the $X$ and $Y$-coordinate of the control point $\left(x_{1}, x_{2}\right)$, respectively.

\subsection{Design Constraints}

Seven design constraints are defined in Table 1 for the input CAD models that represents relationship between design parameters. $\varphi_{1}, \varphi_{2}, \ldots, \varphi_{6}$ are the inequality constraints and $\varphi_{7}$ is the equality constraint. $\varphi_{7}$ is a nonlinear and maintains the $G^{1}$ continuity between the curves at their connection point. $\vec{v}_{1}$ is the vector between control points $\left(x_{5}, x_{6}\right)$ and $\left(x_{7}, x_{8}\right)$, and $\vec{v}_{2}$ is the vector between control points $\left(x_{7}, x_{8}\right)$ and $\left(x_{9}, x_{10}\right) . \theta$ is the angle in radian between vector $\vec{v}_{1}$ and $\vec{v}_{2}$. If $\theta$ is zero, the connecting polygon segments of curve- 1 and curve- 2 are collinear so that there is a $G^{1}$ continuity. Note that determination of the design constraints should be carefully done. Lower and upper bounds of each design parameter specify the length of dimensions of the design space which are shown in Table 2. 
Table 1. Design constraints for the input CAD models

\begin{tabular}{|c|c|}
\hline \multicolumn{3}{|c|}{ Design Constraints } \\
\hline$\varphi_{1}=x_{2}-x_{4}>0$ & $\varphi_{4}=x_{8}-x_{10}>0$ \\
\hline$\varphi_{2}=x_{4}-x_{6}>0$ & $\varphi_{5}=x_{10}-x_{12}>0$ \\
\hline$\varphi_{3}=x_{6}-x_{8}>0$ & $\varphi_{6}=x_{12}-x_{14}>0$ \\
\hline \multicolumn{2}{|c|}{$\varphi_{7}=\theta\left(\vec{v}_{1}, \vec{v}_{2}\right)=0$} \\
\hline
\end{tabular}

Table 2. Lower and upper bounds of design parameters of input CAD models

\begin{tabular}{|c|c|c|c|}
\hline \multicolumn{5}{|c|}{ Vase input design } \\
\hline $0.50 \leq x_{1} \leq 7.50$ & $0.23 \leq x_{5} \leq 6.74$ & $0.14 \leq x_{9} \leq 6.14$ & $0 \leq x_{13} \leq 0.50$ \\
\hline $7.75 \leq x_{2} \leq 9.95$ & $3.00 \leq x_{6} \leq 4.62$ & $0.25 \leq x_{10} \leq 1.75$ & $0 \leq x_{14} \leq 0.70$ \\
\hline $0.50 \leq x_{3} \leq 7.50$ & $0.30 \leq x_{7} \leq 6.32$ & $1.25 \leq x_{11} \leq 2.75$ & \\
\hline $4.75 \leq x_{4} \leq 7.25$ & $1.70 \leq x_{8} \leq 3.00$ & $0 \leq x_{12} \leq 0.80$ & \\
\hline \multicolumn{5}{|c|}{ Glass input design } \\
\hline $0.50 \leq x_{1} \leq 7.5$ & $0.25 \leq x_{5} \leq 6.25$ & $0.04 \leq x_{9} \leq 6.50$ & $0.50 \leq x_{13} \leq 3.50$ \\
\hline $8.25 \leq x_{2} \leq 10.75$ & $5.43 \leq x_{6} \leq 8.28$ & $3.30 \leq x_{10} \leq 6.30$ & $0.25 \leq x_{14} \leq 2.75$ \\
\hline $0.5 \leq x_{3} \leq 7.5$ & $0.5 \leq x_{7} \leq 6.50$ & $0.04 \leq x_{11} \leq 6.50$ & \\
\hline $7.30 \leq x_{4} \leq 9.80$ & $5.76 \leq x_{8} \leq 8.50$ & $1.16 \leq x_{12} \leq 3.50$ & \\
\hline \multicolumn{5}{|c|}{ Bowl input design } \\
\hline $2.15 \leq x_{1} \leq 10.5$ & $3.08 \leq x_{5} \leq 13.0$ & $0.87 \leq x_{9} \leq 8.87$ & $0 \leq x_{13} \leq 1.35$ \\
\hline $11.25 \leq x_{2} \leq 14.75$ & $8.14 \leq x_{6} \leq 11.64$ & $3.60 \leq x_{10} \leq 7.15$ & $0.60 \leq x_{14} \leq 1.75$ \\
\hline $0.45 \leq x_{3} \leq 12.45$ & $2.50 \leq x_{7} \leq 11.50$ & $1.50 \leq x_{11} \leq 10.5$ & \\
\hline $10.30 \leq x_{4} \leq 13.45$ & $6.71 \leq x_{8} \leq 9.29$ & $0.75 \leq x_{12} \leq 3.25$ & \\
\hline
\end{tabular}

\section{RESULTS AND DISCUSSIONS}

Figure 6 shows the test results obtained after inputting cup, glass and bowl models for $N=200$ and $\mu=0.5$ settings. The number of designs obtained after the single run of the ELHS algorithm are eight for the vase, 12 for the glass and seven for the bowl models. And the number of designs obtained by the multiple runs of ELHS algorithm are 223 for the vase, 293 for the glass and 169 for the bowl models. Naturally, greater number of designs are obtained for the multiple runs of the ELHS algorithm. The difference in the number of obtained designs for each input model is due to two factors. First, each input model has different design features, and secondly, the value ranges for the design parameters differs according to each input model. Notice that in Figure 6, distinct designs are obtained from both single run and multiple runs of the ELHS algorithm. The $G^{0}$ and $G^{1}$ continuity is maintain at the connection points of curve- 1 and curve- 2 of the obtained designs.

Parameter Setting: Number of designs generated depends on the number of divisions of the design space. Figure 7 (a) shows the number of designs obtained for the vase model when using different values of $N$. Results here are obtained using the single run of the ELHS algorithm. For $N=200$, eight designs are obtained and 14 designs are obtained for the $N=2000$ setting. The maximum number of designs are obtained for $N=1200$ and remains constant for $N=1700$.

The similarity threshold $\boldsymbol{\mu}$ is taken as 0.5 in the experiments that are shown in Figure 6. Note that diagonal of the scaled design space generated for the test models is $\sqrt{\mathbf{1 4}}$. With the increase in $\boldsymbol{\mu}$, number of designs obtained decreases but distinction between these designs increases. Table 4 shows results for $\boldsymbol{\mu}=\mathbf{0 . 5}$ and $\boldsymbol{\mu}=\mathbf{1 . 0}$ settings when $\boldsymbol{N}=\mathbf{2 0 0}$. For $\boldsymbol{\mu}=\mathbf{0 . 5}, 226$ designs are obtained for the vase model when multiple runs of the ELHS algorithm is applied. And for $\boldsymbol{\mu}=\mathbf{1 . 0}$, only two designs are obtained. 
Khan and Günpınar/ Anadolu Univ. J. of Sci. and Technology A-Appl. Sci. and Eng. 18 (2) - 2017

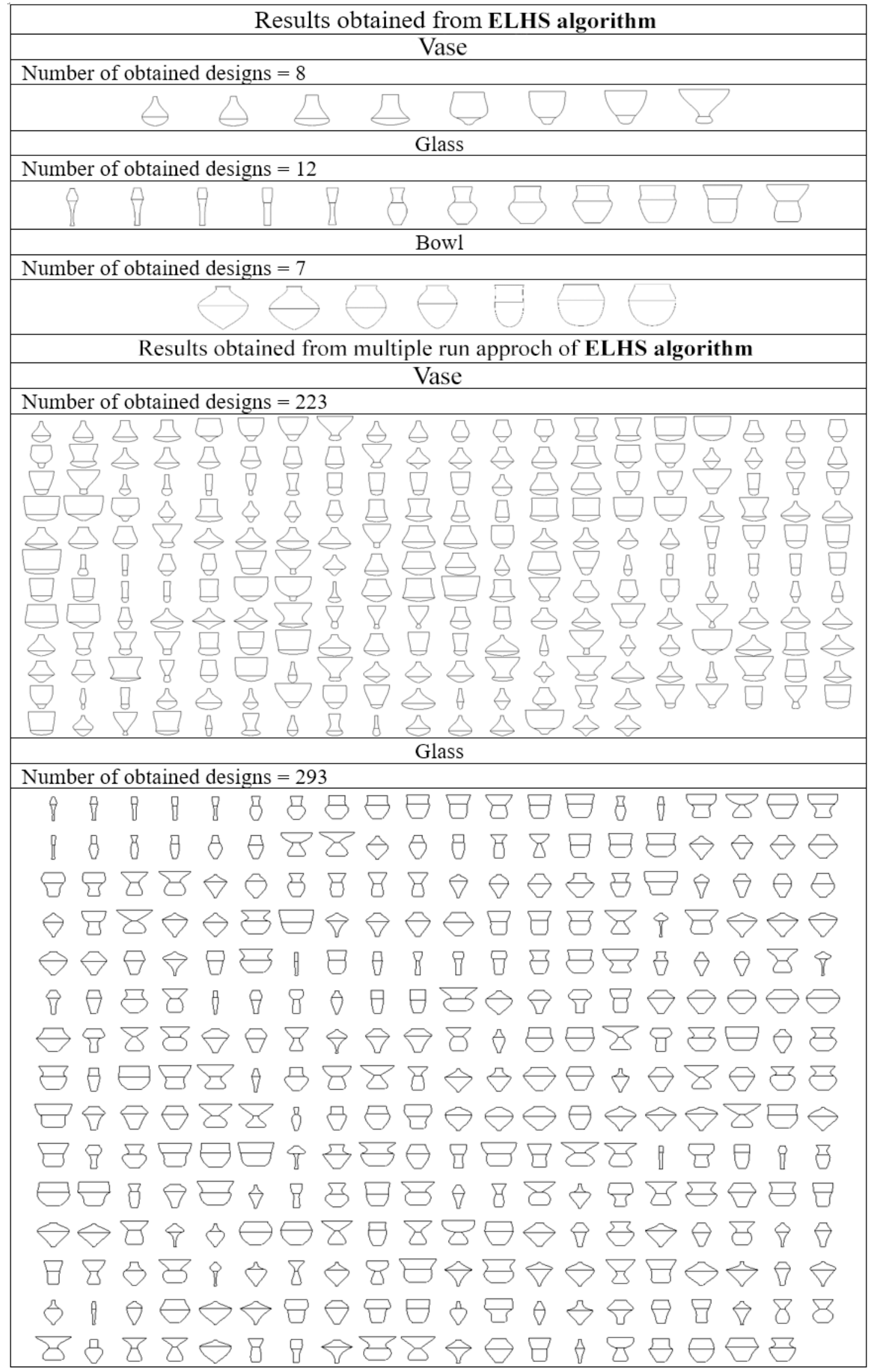




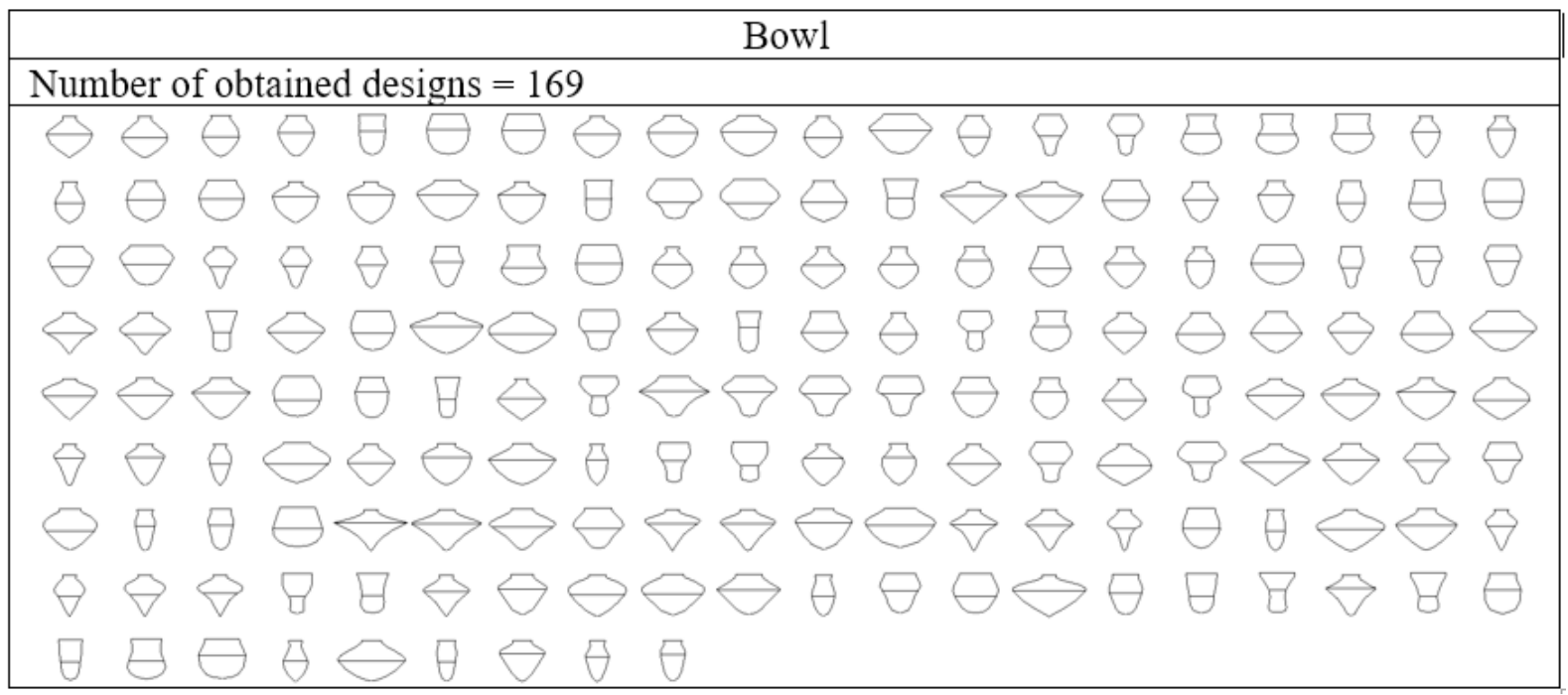

Figure 6. Designs obtained by running the ELHS algorithm single and multiple times $(N=200$ and $\mu=0.5)$

(a)

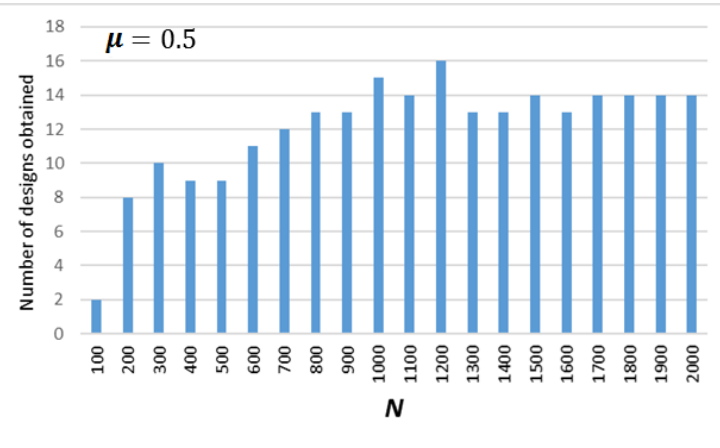

(b)

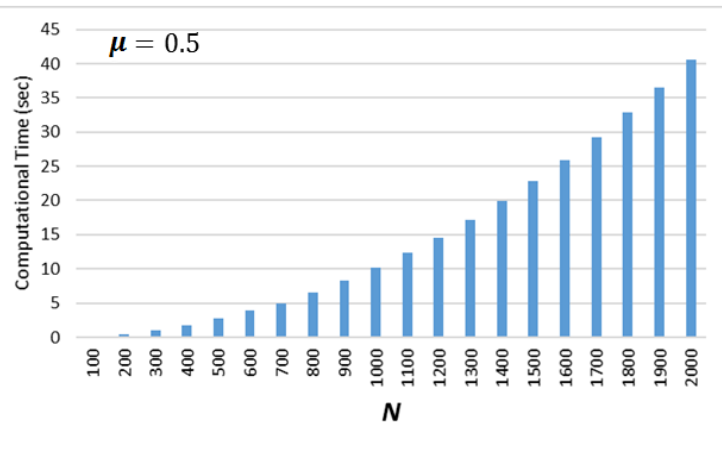

Figure 7. (a) Graph between number of designs obtained and number of design space divisions $(\boldsymbol{N})$ for vase model inputted to ELHS algorithm. (b) Graph between Computational Time and $\boldsymbol{N}$ for vase model inputted to ELHS algorithm

Computational Time: A PC having the Intel Core i7 6700, $3.4 \mathrm{GHz}$ processor and 16 GB memory is used for the experiments in this study. It is observed that computational time increases as the value of $N$ increases. Table 3 shows the processing time of the results provided in Figure 6. When $N$ is high, number of trials to be performed is also high and more number of candidate positions are available to enumerate in each trial. The computation for different values of $N$ for single run of the ELHS algorithm is shown in Figure 7 (b).

Table 3. Computational time of the results provided in Figure 6

\begin{tabular}{|c|c|c|c|}
\cline { 2 - 3 } \multicolumn{1}{c|}{} & \multicolumn{2}{|c|}{ Single run of ELHS } & \multicolumn{2}{c|}{ Multiple runs of ELHS } \\
\cline { 2 - 3 } \multicolumn{1}{c|}{$N=200$} & $\mu=0.5$ & $N=200$ & $\mu=0.5$ \\
\hline Input Design & \multicolumn{2}{|c|}{ Computational Time (sec) } & Computational Time (sec) \\
\hline Vase & \multicolumn{2}{|c|}{0.547} & \multicolumn{2}{|c|}{134} \\
\hline Glass & \multicolumn{2}{|c|}{206} \\
\hline Bowl & 0.453 & 81.5 \\
\hline
\end{tabular}


However, processing time decreases when $\mu$ is closer to 1 and increases when $\mu$ is closer to 0 for multiple runs of the ELHS algorithm. This is because of the fact that total number of runs depends on the size of the design array. When value of $\mu$ is high, less designs are obtained in each run. And less number of runs are performed which results in less processing time. Table 4 shows computational times for $\mu=$ 0.5 and $\mu=1.0$ when vase model is inputted to ELHS algorithm. Multiple runs of the algorithm are done. In table 4, processing time is 0.585 minutes and number of obtained designs are 226 for the $N=$ 100 and $\mu=0.5$ settings. It means there are 266 number of runs are performed. On the other hand, processing time is 0.00571 minutes for $N=100$ and $\mu=1.0$ because only two algorithm runs are performed.

Table 4: Computational time and number of obtained designs for $\mu=0.5$ and $\mu=1.0$

\begin{tabular}{|c|c|c|c|c|}
\cline { 2 - 5 } \multicolumn{1}{c|}{} & \multicolumn{2}{c|}{$\mu=0.5$} & \multicolumn{2}{c|}{$\mu=1.0$} \\
\hline$N$ & $\begin{array}{c}\text { Conputational } \\
\text { Time (minutes) }\end{array}$ & $\begin{array}{c}\text { Number of } \\
\text { designs } \\
\text { obtained }\end{array}$ & $\begin{array}{c}\text { Conputational } \\
\text { Time (minutes) }\end{array}$ & $\begin{array}{c}\text { Number of } \\
\text { designs } \\
\text { obtained }\end{array}$ \\
\hline 100 & 0.585 & 226 & 0.00571 & 2 \\
\hline 200 & 2.2 & 235 & 0.07 & 7 \\
\hline 300 & 5.541 & 257 & 0.23 & 12 \\
\hline 400 & 10.23 & 276 & 0.321 & 11 \\
\hline 500 & 15.34 & 272 & 0.46 & 10 \\
\hline
\end{tabular}

Distribution of obtained designs in the Scaled Design Space: In order to verify similarity between obtained designs Multidimensional scaling (MDS) is used [21]. The parameter values of designs in the design array $(T)$ are scaled between 0 to 1 , and plotted on the 2-dimensional graph as shown in Figure 8. The red points in the graphs represents designs and line between two black points indicates the diagonal of the scaled space. The closeness of two point indicated their geometrical similarity. In Figure 8, image (a) and (b) and has better distributions of designs than image (c). Image (c) shows that in case of the bowl as input model, designs are not selected from the entire design space. This can occur because of different reasons, mainly: the empty region could be an infeasible region in the design space or the designs in this region may not satisfy the similarity constraints and get eliminated.

(a)

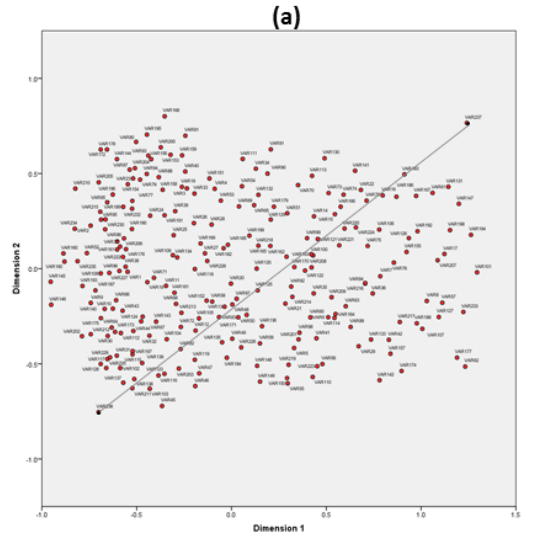

(b)

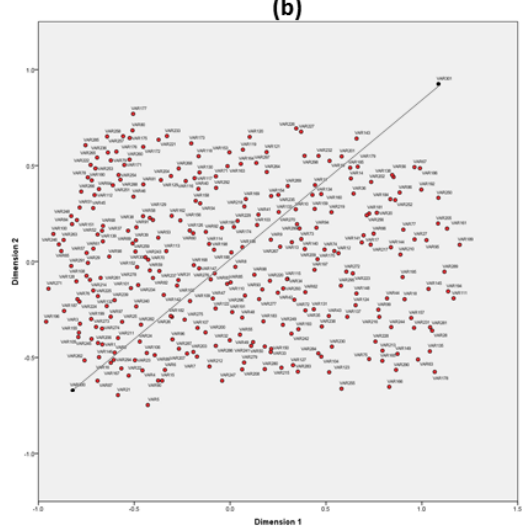

(c)

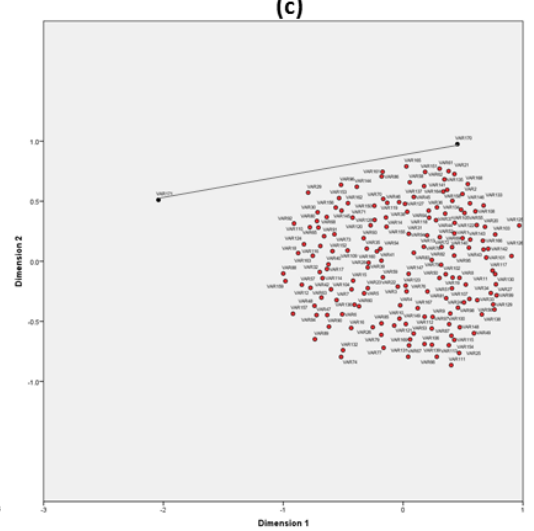

Figure 8. Multidimensional scaling of designs obtained by inputting (a) Vase model (b) Glass model and (c) Bowl model in order to observe the similarity 
Limitation: A sampling technique is considered to have good space filling property, if the sampled designs are uniformly distributed throughout the design space. The designs obtained by ELHS does not guarantee the space filling property as it depends on the input design. However, the objective of current research to provide designers with a variety of design options for their product within the product's design space is achieved.

\section{CONCLUSIONS AND FUTURE WORKS}

Latin Hypercube Sampling technique is extended in order to perform sampling in the high dimensional constrained design space with equality and inequality constraints. Designs space is formed by the designs parameters, designs constraints and lower/upper bounds for each design parameter. Each dimension of design space is divided into certain number of intervals to start the sampling process. The LH-rule is considered during sampling. ELHS starts the sampling process by performing enumeration during the trials. In every trial, all the candidate positons of each design parameter are enumerated and the one which minimizes the cost is selected. Designs with similar geometries are eliminated on the basis of similarity constraint and design with variant geometries are obtained.

As a future work, authors of current research will try to further improve the space filling property of the ELHS algorithm. Methods for the iterative improvements [22] will be studied and implemented in order to polish the sampling quality. Enumeration based operators can also be proposed for better uniformity of sampled designs. Other sampling techniques such as centroidal Voronoi tessellation and Latinization [23] will also be studied in order to check if these techniques can provide improved results.

\section{ACKNOWLEGMENT}

The authors would like to pay their deepest gratitude to the Scientific and Technological Research Council of Turkey (TÜBİTAK) for sponsoring this project (Project Number: 214M333).

\section{REFERENCES}

[1] MaKa M, Beckman R and Conover W. A comparision of three methods for selecting values of input variables in the analysis of output from computer code. Technimeterics 1979; 42: 55-61.

[2] Iman R, Conover W. Small sample sensitivity analysis techniques for computers models, with an application to risk assessment. Communications in statistics-theory and methods 1980; 9: 1749-1842.

[3] Johson R, Wichern D. Applied Multivariate Statistical Analysis. New Jersey. Prentice-Hall, 2002. pp. 767.

[4] Morris M, Mitchell T. Exploratory designs for computational experiments. Journal of Statistical Planning and Inference 1995; 43: 381-402.

[5] Deutsch J, Deutsch C. Latin hypercube sampling with multidimensional uniformity. Journal of Statistical Planning and Inference 2012; 142: 763-772.

[6] Cioppa T, Lucas T. Efficient nearly orthogonal and space-filling latin hypercubes. Technometrics 2007; 49: 45-55.

[7] Qian P. Sliced Latin Hypercube Designs. Journal of the American Statistical Association 2012; 107: 393-399. 
Khan and Günpınar/ Anadolu Univ. J. of Sci. and Technology A-Appl. Sci. and Eng. 18 (2) - 2017

[8] Cao RY, Liu MQ. Construction of second-order orthogonal sliced Latin hypercube designs. Journal of Complexity 2014; 30: 762-772.

[9] Prescott P. Orthogonal-column Latin hypercube designs with small samples. Computational Statistics and Data Analysis 2009; 53: 1191-1200.

[10] Sacks J, Schiller S, Welch W. Designs for computer experiments. Technometrics 1989; 31: 41-47.

[11] Bates S, Sienz J, Langley D. Formulation of the Audze-Eglais uniform Latin hypercube design of experiments. Advances in Engineering Software 2003; 34: 493-506.

[12] Bates S, Sienz J, Toropov V. Formulation of the optimal latin hypercube design. In: 45th AIAA/ASME/ASCE/AHS/ASC Structures, Structural Dynamics \& Materials Conference; 19-22 April 2004; Palm Springs, California.

[13] Audze P, Eglais V. New approach for planning out of experiments. Problems Dynam Strengths 1977; 35: 104-107.

[14] Rajabi M, Ashtiani B and Janssen H. Efficiency enhancement of optimized Latin hypercube sampling strategies: Application to Monte Carlo uncertainty analysis and meta-modeling. Advances in Water Resources 2015; 76: 127-139.

[15] Leary S, Bhaskar A, Keane A. Optimal orthogonal-array-based latin hypercubes. Journal of Applied Statistics 2003; 30: 585-598.

[16] Joseph V, Hung Y. Orthogonal-maximin latin hypercube designs. Statistica Sinica 2008; 18: 171186.

[17] D. Bingham, R. Sitter, B. Tang. Orthogonal and nearly orthogonal designs for computer experiments. Biometrika 2009; 96: 51-65.

[18] Myšáková E, Lepš M, Kučerová A. A Method for Maximin Constrained Design of Experiments. In: Eighth International Conference on Engineering Computational Technology; 2012: Stirlingshire, Scotland.

[19] Mysakova E, Leps M. Method for Constrained Designs of Experiments in Two Dimensions. in: 18th International Conferance in Enginnering Mechanics; 14-17 May 2012; Svratka, Czech Republic. pp. 901-914.

[20] Fuerle F, Sienz J. Formulation of the Audze-Eglais uniform Latin hypercube design of experiments for constrained design spaces. Advances in Engineering Software 2011; 42: 680-689.

[21] Davison ML. Multidimensional scaling. Krieger Publishing Company.

[22] Liefvendahl M, Stocki R. A study on algorithms for optimization of Latin Hypercubes. Journal of Statistical Planning and inference 2006; 136: 3231-3247.

[23] Hou S. Latinized, Improved LHS, and CVT Point Sets in Hypercubes. International Journal of Numerical Analysis and Modeling 2007; 4: 729-743. 